\title{
Opto-electronic cross-phase tuneable system based on cascaded intensity modulators
}

\author{
Marc Sans, Cyril Renaud and John E. Mitchell \\ Department of Electronic and Electrical Engineering \\ University College London \\ London, UK \\ m.sans@ucl.ac.uk, c.renaud@ucl.ac.uk,j.mitchell@ucl.ac.uk
}

\begin{abstract}
We demonstrate a dual-output microwave photonic system that allows 360-degree per element up-converted phase adjustment with potential applicability in phased array systems. Two self-correlated Ku-band carriers are generated in the optical domain through beating by means of concatenated intensity modulators fed by lower frequency electrical sources, where the phase-tuning originates. The system shows high correlation along the full phase sweep between its outputs, which are characterized individually in terms of phase noise and spectral stability.
\end{abstract}

Keywords-beamforming; microwave photonics; phased-array systems

\section{INTRODUCTION}

Since the first deployments of phased array antenna (PAA) systems based on distributed radar to detect intrusive aircrafts during the World War II, the PAA field has evolved technologically and expanded in terms of applications vastly [1-7]. Sectors such as telecommunications (ground and satellite), astronomy, health care and automotive have adopted phased array antenna technology - based on analogue and/or digital means - for a wide range of frequency bands and user case scenarios.

Nowadays, beamforming systems are used not only to augment antenna's directivity and steer its main lobe, but also for more complex capabilities such as beam nulling (interference avoidance) or multi-beam services [8]. In order to accomplish these functions, each antenna element requires a synchronised feeding signal with a different amplitude and temporal deviations (weighting). For the later, phase shifting (PS) elements are traditionally used as an approximation to time delay for narrowband systems, in order to reduce system's complexity and will also be assumed in this work.

Particularly, for next generation wireless communications systems, an ever increasing capacity demand ultimately translates into better frequency use and/or larger bandwidth allocation. Due to spectrum congestion below $6 \mathrm{GHz}$ bands, upper spectrum regions such as: millimetre-wave (30-300 $\mathrm{GHz})$, terahertz $(0.3-3 \mathrm{THz})$ and free-space optical have been receiving increased interest over the last years for future wireless communication systems [9-12]. Usage of those bands imply higher path loss and absorption where line-of-sight (LoS) traditionally becomes a must to account for a positive link margin. PAA systems with beamforming capabilities are a potential solution to aforementioned challenges.

Microwave photonics aims to translate the inherent benefits of optical systems (mostly, large bandwidth and low distribution losses) into the microwave domain. Several functionalities such as: signal generation, processing, filtering and sampling have been demonstrated already [13-15]. A variety of work has been carried out for beamforming solutions with further benefits such as broad tuneability and squint free steering for wideband signals. Technologies such as: voltagecontrolled phase-shifters, fibre based grating structures with tuneable lasers, liquid-crystal systems or optical ring resonators have been demonstrated [16-19]. Furthermore, those systems which can potentially be integrated on-chip are of especial interest for market feasibility due to a promise in reduction of: size, weight, power consumption and cost.

We present a system which accomplishes microwave photonic signal generation while up-converting a lowfrequency phase tuneable signal to a higher-frequency band. The system benefits from lack of use of high frequency phase shifting elements and provides high frequency tuneability. This paper is organized as follows: in Section I we have introduced the topic and need; Section II presents the microwave photonic cross-phase tuneable concept including a simulation scenario; next, in Section III we discuss the experimental setup proving the concept; finally Section IV concludes our work.

\section{CONCEPT PROPOSAL}

Frequency translation through mixing is a well-known method used across the radio community to minimize the need of higher frequency components for technological and cost reasons. Still in the analogue domain, the concept of intermediate frequency beamforming is also employed. We propose the use of up/down-conversion within a microwave photonics system for phase distributed systems targeted at $5 \mathrm{G}$ delivery networks [20]. 

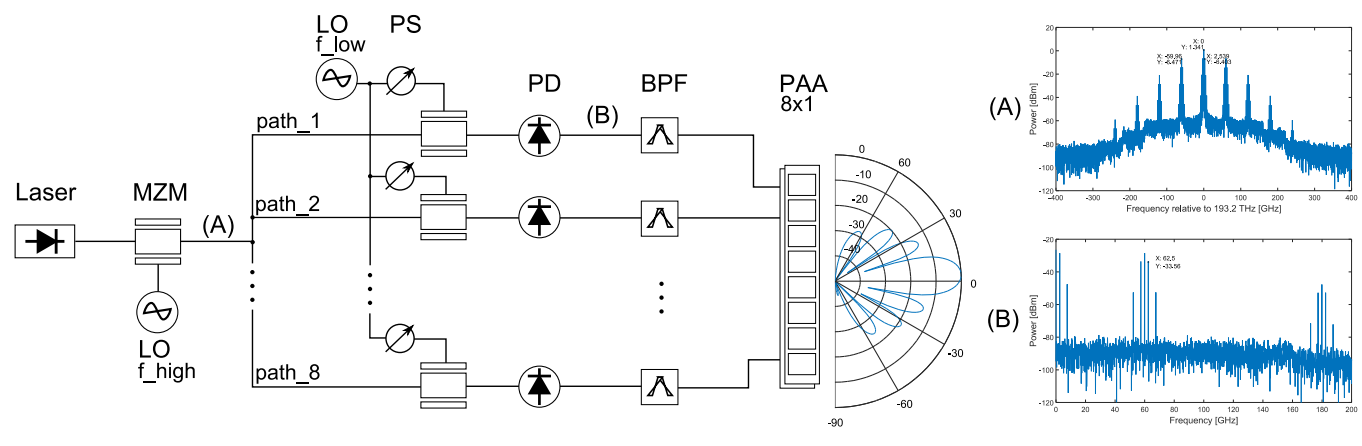

Fig. 1. Simulation diagram

In our conceptual system (Fig. 1), we generate several correlated optical tones with a frequency spacing of $f$ high using a single laser source and a high frequency intensity modulator. The output signal is then equally split across several paths. Each of the outputs is then individually fed onto a lower frequency intensity modulator. At the same time, those lower frequency intensity modulators are fed with a phase-adjustable $f$ low tone. The distribution of the low frequency sources is synchronized. A photodiode (PD) follows each arm and downconverts to the electrical domain all beating tones originating from the concatenated optical modulations. A band-pass filter (BPF) per arm is included after each photodiode to select the frequency of interest, $f$ final $=f$ high $+f_{-}$low.

The proposed concept for $\lambda=1550 \mathrm{~nm}$ with local oscillator (LO) frequencies satisfying a radiation $\left(f_{-}\right.$final $)$frequency in the $\mathrm{Ku}, \mathrm{Ka}$ and millimetre-wave $(60 \mathrm{GHz})$ bands has been simulated (notice the change in centre frequency of the BPF) showing a linear phase response and broad frequency tuneability. VPI Transmission Maker has been used with single electrode Mach-Zehnder Modulators (MZMs) as amplitude modulators. From the simulation, we obtain the optimum DC bias operation points of the MZMs (aka, maximal accumulated electrical power at the output of the system) as shown in Fig. 2. The obtained output signals are interfaced with Matlab to illustrate the beamforming concept in an $8 \times 1$ uniform linear array (see Fig. 3). The element pattern is approximated by a cosine function (factor of 1.35), and the spacing between elements is set to $\lambda / 2$ (different for aforementioned $f$ final). No amplitude tapering is used.

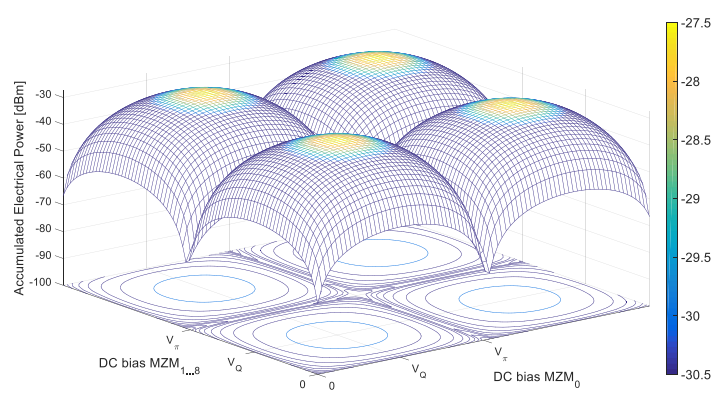

Fig. 2. MZM operation points (DC bias)

\section{LABORATORY DEMONSTRATOR}

A proof of concept of the cross-phase tuneable system based on cascaded single-arm Mach-Zehnder Modulators is illustrated in Fig. 4. Relevant power measurements (in parentheses) across the setup are included there for better comprehension. The objective of the setup is to up-convert the phase information of two $3 \mathrm{GHz}$ sources (one source per arm) onto $13.5 \mathrm{GHz}$ tones such as the phase-induced difference between them is kept correlated.

First, 3 tones at $1554 \mathrm{~nm}$ spaced $10.5 \mathrm{GHz}$ are generated using a 40 Gbps MZM (DC bias adjusted such carrier-tosidebands power difference is minimized) with a $10 \mathrm{KHz}$ intrinsic linewidth external cavity laser. The polarization state is manually adjusted prior to each MZM through polarization controllers (PC) for polarization alignment. The $40 \mathrm{Gbps}$ MZM output tones are then amplified using a double stage erbium doped fibre amplifier (EDFA: Noise figure $=6.25 \mathrm{~dB}$ ) to combat power losses across the setup. The optically amplified signal is then routed onto a $4 \mathrm{~nm}$ bandwidth tuneable optical bandpass filter to reduce the Amplified Spontaneous Emission (ASE) noise. Then, the optical signal is equally split and used as the input to 10 Gbps MZMs per arm (both with similar specifications). Each $10 \mathrm{Gbps}$ MZM is driven through a different signal generator $(f=3 \mathrm{GHz}$, P.out $=10 \mathrm{dBm})$ and referenced using a $10 \mathrm{MHz}$ internal local oscillator. These signal generators provide the adjustment of phase and amplitude individually onto each arm. The $10 \mathrm{Gbps}$ modulators are biased at their quadrature points as per simulation results shown in previous section.

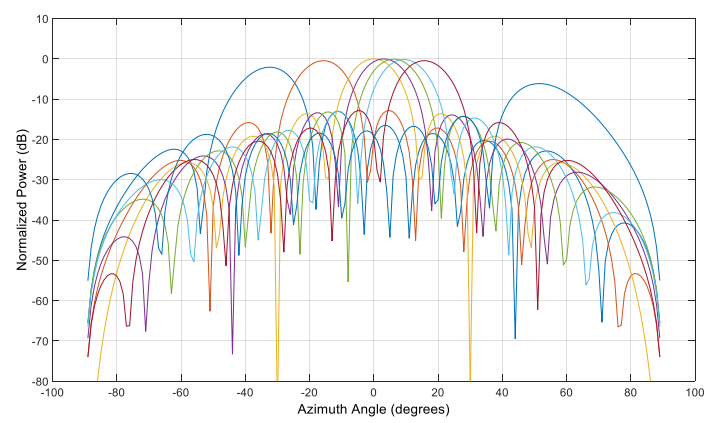

Fig. 3. Far field radiation pattern for -30 to +50 degrees scan 


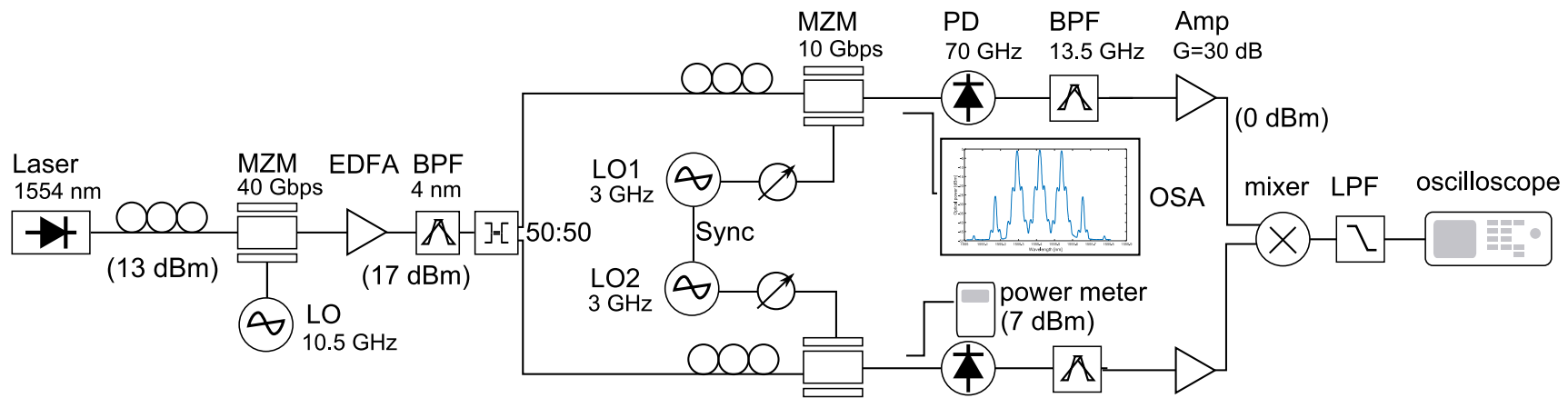

Fig. 4. Laboratory diagram

The optical spectrum measurements are obtained with a $0.01 \mathrm{~nm}$ resolution optical spectrum analyser (OSA). Before each photodiode, 3 higher peaks (10.5 GHz spaced) $0 \mathrm{dBm}$ are amplitude modulated $(3 \mathrm{GHz})$ resulting on $-20 \mathrm{dBm}$ tones. The noise floor around the band of interest after optical amplification is $-50 \mathrm{dBm}$. Optical average power prior to each photodiode is kept around $7 \mathrm{dBm}$.

A pair of $70 \mathrm{GHz}$ photodiodes are used to down-convert all beating tones. Afterwards, a band-pass filter (BPF) $\left(f_{c}=13.5\right.$ $\mathrm{GHz}, \mathrm{BW} 3 \mathrm{~dB}=140 \mathrm{MHz}$ ) is used to select those of interest, and a double-amplification stage (total gain $\sim 30 \mathrm{~dB}$ ) for each arm is used to provide sufficient power to the phase-detector. The phase-detector is composed of a wideband doublebalanced mixer followed by a low-pass filter (LPF) (DC-780 $\mathrm{MHz}$ ). Its DC output will be proportional to the phasedifference between the two input ports assuming amplitude stability onto the input ports. Although mixer's recommended operation point is an LO input of $13 \mathrm{dBm}$, a lower power input signal has been observed to perform correctly after characterization at several input levels.

Finally, the filtered output of the mixer is connected to a digital communications analyser for a DC voltage measurement. In order to measure the phase-response, one signal generator is phase-swept across its entire range ( $-\pi$ to $+\pi$ ) while subsequent voltage average measurement from the analyser are read out. The measurement is composed of 126 points captured over a period of 12 seconds.

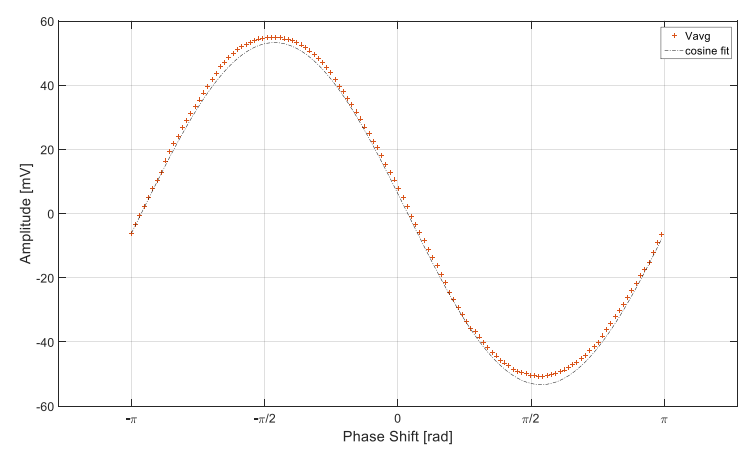

Fig. 5. Response of phase-measurement setup and cosine fit
The response of the phase-measurement system for a 3 $\mathrm{GHz}$ to $13.5 \mathrm{GHz}$ optically up-converted tone is shown in Fig. 5. The characterisation shows a well-balanced function (symmetry across X-axis), a voltage peak to peak of $105 \mathrm{mV}$ and a residual DC offset of $2 \mathrm{mV}$. The graph is matched with a cosine fit showing an accurate phase-difference predictability.

As shown in Fig. 6, selected microwave photonic generation and transport methods enable high frequency stability onto the $13.5 \mathrm{GHz}$ tones regardless of the observed laser source drift over a 3 hours waterfall measurement. Phase noise measurements were also conducted onto the RF sources and after each $13.5 \mathrm{GHz}$ band-pass filter (see Fig. 7). As expected, the output phase noise slightly degrades from the cumulated original sources. Besides thermal and vibration contributions, we also attribute that increase and peaks to imperfections onto optical components and the FC/PC fibre adapters used.

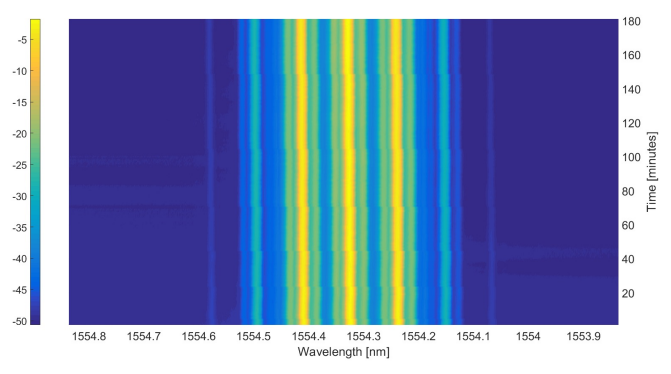

a) Spectrogram prior photodiode

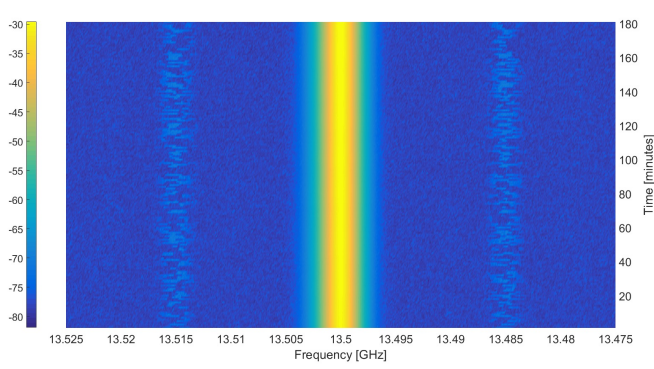

b) Spectrogram after photodiode

Fig. 6. Stability measurement 


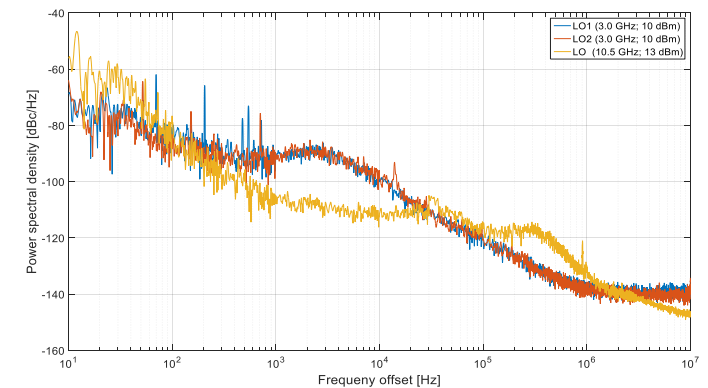

a) Signal generators

Fig. 7. Single side-band phase noise measurement

\section{CONCLUSIONS}

We have presented an opto-electronic beamforming concept with up-conversion phase stage using cascaded optical intensity modulators. The system avoids the use of high frequency phase shifters and enables high frequency tuneability - if tuneable band pass filters at each photodetector output, and careful selection of modulated tones are included.

The proof of concept shows a reliable frequency and phase stability, despite the simplicity of the setup (where any feedback or control loops are utilized) which have been observed over long duration spectral measurements and phase noise measurements. Anticipated power loss across the system mostly due to: conversion stages, optical modulators insertion losses and undesired beating tones have been compensated by use of optical (EDFA) and electrical amplifier stages.

\section{ACKNOWLEDGMENT}

The fist author would like to thank Katarzyna Balakier, Haymen Shams and Luis González from University College London, and Róbert Horváth from Université Savoie Mont Blanc for helpful discussions.

This work was supported by the Initial Training Network, FiWiN5G, funded by the H2020 Marie Curie Actions of the European Commission (H2020-MSCA-ITN-2014 N642355).

\section{REFERENCES}

[1] R. C. Watson, "Radar Origins Worldwide: History of Its Evolution in 13 Nations Through World War II," Trafford Publishing, 2009.

[2] A. Boonstra, M. Garrett, G. Kruithof, M. Wise, A. van Ardenne, J. Yan, J. Wu, J. Zheng, E. K.A. Gill, J. Guo, M. Bentum, J. N. Girard, X. Hong, T. An, H. Falcke, M. Klein-wolt, S. Wu, W. Chen, L. Koopmans, H. Rothkaehl, X. Chen, M. Huang, L. Cheng, L. Gurvits, P. Zarka, B. Cecconi and H. de Hann, "Discovering the sky at the Longest Wavelengths (DSL)" IEEE Aerospace Conference, 2016.

[3] C. Papadacci, M. Pernot, M. Couade, M. Fink and M. Tanter, "Highcontrast ultrafast imaging of the heart," IEEE Transactions on Ultrasonics, Ferroelectrics, and Frequency Control, vol. 61, no. 2, February 2014.

[4] R. Bill and W. Holpp, "Modern phased array radar systems in Germany," IEEE International Symposium on Phased Array Systems and Technology, 2016.

[5] P. Angeletti and M. Lisi, "Multimode Beamforming Networks for Space Applications," IEEE Antennas and Propagation Magazine, , Vol. 56, No. 1 , February 2014.

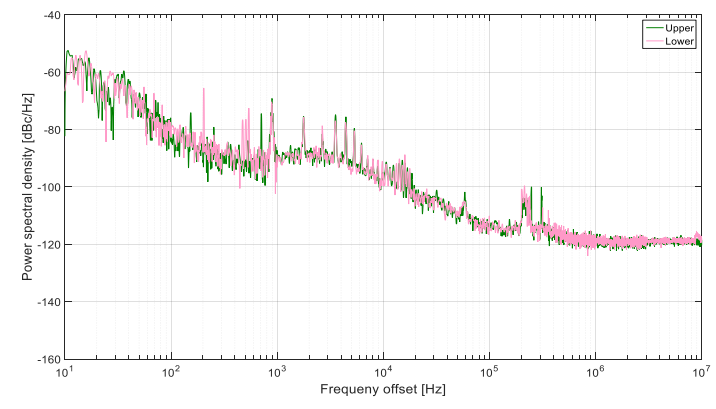

b) After band-pass filters

[6] J. Hasch, E. Topak, R. Schnabel, T. Zwick, R. Weigel and C. Waldschmidt, "Millimeter-wave Technology for Automotive Radar Sensors in the $77 \mathrm{GHz}$ Frequency Band," IEEE Transactions on Microwave Theory and Techniques, vol. 60, no. 3, March 2012.

[7] W. Roh, J. Seol, J. Park, B. Lee, Y. Kim, J. Cho, K.Cheun and F. Aryanfar, "Millimeter-Wave Beamforming as an Enabling Technology for 5G Cellular Communications: Theoretical Feasibility and Prototype Results," IEEE Communications Magazine, February 2014

[8] L. A. Greda and A. Dreherl, "Beamforming capabilities of array-fed reflector antennas," IEEE Antennas and Propagation (EUCAP), Proceedings of the 5th European Conference on, 2011.

[9] Z. Pi and F. Khan, "An Introduction to Millimeter-Wave Mobile Broadband Systems," IEEE Communications Magazine, June 2011.

[10] H. Song and T. Nagatsuma, "Present and Future of Terahertz Communications," IEEE Transactions on terahertz science and technology, vol. 1, no. 1, September 2011.

[11] Z. Sodnik, C. Heese, I. Carnelli, A. Galvez and T. Dreischer, "Multipurpose laser communication system for the asteroid impact mission (AIM)," IEEE International Conference on Space Optical Systems and Applications (ICSOS), 2015.

[12] H. Hemmati, A. Biswas and I. B. Djordjevic, "Deep-Space Optical Communications: Future Perspectives and Applications," Proceedings of the IEEE, vol. 99, no. 11, November 2011

[13] J. Capmany and I. Gasulla "Microwave Photonics," J. Webster (ed.), Wiley Encyclopedia of Electrical and Electronics Engineering, 2016.

[14] S. Iezekiel, M. Burla, J. Klamkin, D. Marpaung and J. Capmany, "RF Engineering Meets Optoelectronics," IEEE Microwave Magazine, pp. 28-45, September 2015

[15] D. Marpaung, C. Roeloffzen, R. Heideman, A. Leinse, S. Sales and J. Capmany, "Integrated microwave photonics," Laser \& Photonics Reviews 7, no. 4, pp. 506-538, 2013.

[16] R. A. Soref, "Voltage-Controlled Optical/RF Phase Shifter," Journal of Lightwave Technology, vol. LT-3, no. 5, pp. 992-998, 1985.

[17] M.A. Piqueras, G. Grosskopf, B. Vidal, J. Herrera, J.M. Martinez, P. Sanchis, V. Polo, Juan.L. Corral, A. Marceaux, J. Galiere, J. Lopez, A. Enard, J.-L. Valard, O. Parillaud, E. Estebe, N. Vodjdani, Moon-Soon Choi, J.H. den Besten, F.M. Soares, M.K. Smit and J. Marti, "Optically Beamformed Beam-Switched Adaptive Antennas for Fixed and Mobile Broad-Band Wireless Access Networks," IEEE Transactions on Microwave Theory and Techniques, vol. 54, no. 2, 2006.

[18] G. Serafino, M. Presi, A. Malacarne, P. Ghelfi, C. Porzi and A. Bogoni, "Simultaneous beam steering of multiple signals based on optical wavelength selective switch," European Radar Conference (EuRAD), pp. 404-407, 2014.

[19] C. G. H. Roeloffzen, P. W. L. van Dijk, R. M. Oldenbeuving, I. Dove and R. B. Timens, "Integrated optical beamformers," IEEE Avionics and Vehicle Fiber-Optics and Photonics Conference (AVFOP), pp. 61-62, 2015.

[20] J. E. Mitchell, "FiWiN5G - FIber-Wireless Integrated Networks for 5th Generation delivery," International Conference on Transparent Optical Networks (ICTON), ISSN 2161-2064, 2016. 
